\title{
14,15-EET induces the infiltration and tumor-promoting function of neutrophils to trigger the growth of minimal dormant metastases
}

\author{
Jing Luo ${ }^{1, *}$, Xin-Xia Feng ${ }^{2, *}$, Chao Luo ${ }^{1}$, Yu Wang ${ }^{1}$, Dong Li ${ }^{1}$, Yu Shu ${ }^{1}$, Shan-Shan \\ Wang $^{1}$, Jian Qin ${ }^{1}$, Yong-Chao Li ${ }^{1}$, Jiu-Ming Zou ${ }^{1}$, De-An Tian ${ }^{2}$, Gui-Mei Zhang ${ }^{1}$, \\ Zuo-Hua Feng ${ }^{1}$ \\ ${ }^{1}$ Department of Biochemistry and Molecular Biology, Wuhan University, School of Basic Medicine, Hubei, Wuhan 430030, \\ People's Republic of China \\ ${ }^{2}$ Tongji Hospital, Tongji Medical College, Huazhong University of Science and Technology, Hongshan, Wuhan 430030, People's \\ Republic of China \\ *These authors contributed equally to this work
}

Correspondence to: Zuo-Hua Feng, email: fengzhg_tj@163.com Gui-Mei Zhang, email: zhanggm58@163.com

Keywords: neutrophils, recruitment, metastases, dormancy, epoxyeicosatrienoic acids

Received: January 06, 2016

Accepted: May 13, 2016

Published: May 30, 2016

\section{ABSTRACT}

Infiltrating neutrophils are known to promote in the development of tumor. However, it is unclear whether and how neutrophils are involved in triggering the growth of dormant metastases. Here we show that 14,15-epoxyeicosatrienoic acid (14,15-EET) can trigger the growth of dormant micrometastases by inducing neutrophilic infiltration and converting neutrophil function. 14,15-EET triggered neutrophil infiltration in metastatic lesions by activating STAT3 and JNK pathways to induce the expression of human IL-8 and murine CXCL15 in corresponding tumor cells. The continuous expression of hIL-8/mCXCL15 was maintained by the sustained and enhanced activation of JNK pathway. 14,15-EET up-regulated miR-155 expression by activating STAT3 and JNK pathways. miR-155 in turn down-regulated the expression of SHIP1 and DET1, thus augmenting the activation of JNK and C-Jun. Moreover, the function of neutrophils was converted from tumor-suppressing to tumor-promoting by 14,15-EET in vivo. By inducing the production of G-CSF/IL-6 in vivo, 14,15-EET induced the enhancement of STAT3 activation in neutrophils to increase MMP-9 expression and decrease TRAIL expression. Neutrophil-derived MMP-9 was required for 14,15-EET to induce angiogenesis during the growth of dormant micrometastases. Depleting neutrophils or inhibiting hIL-8/ mCXCL15 up-regulation resulted in the failure of 14,15-EET to promote the development of micrometastases. These findings reveal a mechanism through which the infiltration and tumor-promoting function of neutrophils could be induced to trigger the growth of dormant metastases, which might be a driving force for the tumor recurrence based on dormant metastases.

\section{INTRODUCTION}

The minimal dormant metastases have been recognized as the main cause of cancer recurrence [1-3]. Tumor dormancy is maintained in the microenvironment unfavorable for tumor cell proliferation [3, 4]. When microenvironmental conditions shift to support tumor expansion, dormant tumors can resume active growth and progression [3]. The failure of angiogenesis is a factor that contributes to the maintenance of the dormant state $[1,5]$. Accordingly, the activation of angiogenesis has been implicated as a trigger for the initiation of growth by dormant tumor cells $[1,5]$. However, the mechanisms underlying the angiogenesis during the growth of dormant metastases remain poorly understood.

Polymorphonuclear leukocytes (PMNs or neutrophils) can produce matrix metalloproteinase-9 (MMP-9) to initiate angiogenesis in the development of tumor [6]. The potent angiogenic factors such as VEGF and FGF2 are usually sequestered in an inactivated form to the extracellular matrix (ECM) [7]. MMP-9 remodels ECM and releases VEGF and FGF2, which 
then act on endothelial cells to prompt re-vascularization [7]. Although many types of cells can produce MMP-9 [3], infiltrating neutrophils have been found to trigger angiogenesis in the development of tumor $[6,8]$, and support metastatic initiation $[9,10]$. On the other hand, naive neutrophils have the capacity to suppress angiogenesis by secreting neutrophil elastase (NE) to degrade VEGF and FGF2, and releasing TRAIL to induce vascular disruption [7, 11]. During tumor progression, neutrophil function is converted from tumor-suppressing to tumor-promoting $[12,13]$, thus producing more MMP-9 but releasing much less NE and TRAIL [12]. However, despite clear evidence that neutrophils promote angiogenesis during tumor development, the relationship between neutrophils and dormant metastases remains unclear. The failure of angiogenesis in dormant metastases may hint at the absence of neutrophils in these metastatic lesions. So far little is known about whether and how neutrophils are recruited to the dormant metastases to initiate angiogenesis.

14,15-epoxyeicosatrienoic acid (14,15-EET) is one of four regioisomeric EETs (5,6-EET, 8,9EET, 11,12-EET, and 14,15-EET) that correlate with various biological processes, including vasorelaxation, inflammation, and the response to tissue injury [14]. In recent years, however, 14,15-EET has been found to promote tumor cell proliferation, the growth of primary tumors, and tumor metastasis [14-16]. 14,15-EET can also stimulate the endothelial cells to express VEGF and FGF2 $[17,18]$. These findings raised the question of whether the dormant metastases might be influenced by the elevation of the circulating 14,15-EET in absence of primary tumor, since EET levels can be directly influenced by nutrients and inflammatory processes $[14,15]$. Here, we show that 14,15 -EET could induce neutrophilic infiltration in metastatic lesions and the conversion of neutrophil function, thus triggering the growth of minimal dormant metastases. Neutrophil-derived MMP-9 is required for 14,15 -EET to induce angiogenesis during the growth of dormant metastases. In neutrophil-depleted mice or in MMP-9/- mice, 14,15-EET failed to induce angiogenesis in metastatic lesions and metastases development.

\section{RESULTS}

\section{Growth of minimal dormant metastases can be triggered by 14,15-EET}

Non-metastatic tumor cells could acquire the invasive capacity after stimulation by extracellular signal molecules. However, these cells could only form the dormant micrometastases, but not macroscopic metastases, after extravasation $[19,20]$. To determine whether 14,15-EET could trigger the growth of dormant micrometastases, we inoculated the mice with non-metastatic tumor cells (B16F0, HepG2, and MCF-7 cells) that were pretreated with TGF- $\beta 1 /$
$\mathrm{H}_{2} \mathrm{O}_{2} / \mathrm{HOCl}(\mathrm{T} / \mathrm{H} / \mathrm{H})$ to induce the invasive capacity, and treated the mice with 14,15-EET (Supplementary Figure $\mathrm{S} 1$ ). The growth of dormant micrometastases was triggered by 14,15-EET (Figure 1A, 1B, and Supplementary Figure $\mathrm{S} 2 \mathrm{~A}$ ), resulting in the formation of visible metastatic nodules on the surface of lung (Figure. 1C, 1D, and Supplementary Figure S2B). 14,15-EET promoted the growth of metastases in a dose-dependent manner (Supplementary Figure S2C). The other three regioisomers of EET were much less effective (Supplementary Figure S2D). Furthermore, the effect of 14,15-EET was abolished by 14,15-EEZE, an antagonist of 14,15-EET [14], but was not mimicked or influenced by 14,15-DHET, the metabolite of 14,15-EET [14] (Supplementary Figure S2E).

\section{4,15-EET-induced neutrophilic infiltration is required for the growth of dormant metastases}

To clarify whether neutrophils might be involved in 14,15-EET-induced growth of dormant metastases, we detected the infiltration of neutrophils in the lung tissues harboring dormant metastases (B16F0 model) by detecting the mRNAs of CD11b and Ly6G (Figure 2A), immunohistochemical staining (Figure 2B), and immunofluorescence (Supplementary Figure S3A). Neutrophils were not observed in the lung tissues harboring dormant metastases. The treatment with 14,15 -EET induced the infiltration of neutrophils in the metastatic lesions, but not in control lung tissues. To ascertain the role of neutrophils in 14,15-EET-induced growth of dormant metastases, we depleted neutrophils in vivo (Supplementary Figure S3B). Neutrophil depletion abrogated the promoting effect of 14,15-EET on the development of metastatic lesions into visible metastatic nodules in the metastatic models using B16F0 cells (Figure 2C), HepG2 cells and MCF-7 cells (Supplementary Figure S3C). Similarly, depleting neutrophils also suppressed the promoting effect of 14,15-EET on the metastatic growth of B16F1 cells, a metastatic clone of melanoma B16 (Supplementary Figure S3D). These results suggested that 14,15 -EET induced neutrophilic infiltration to promote the development of metastatic lesions.

\section{4,15-EET promotes hIL-8/mCXCL15 expression in tumor cells to recruit neutrophils}

To analyze the mechanisms underlying the recruitment of neutrophils during the growth of dormant metastases, we investigated whether 14,15EET might induce the production of neutrophil chemoattractants by detecting the expression of the genes coding for CXCL1, CXCL2, CXCL5, CXCL15, CCL2, CCL3, CCL4, CCL5, GM-CSF [21-23]. The results showed that 14,15-EET substantially increased the expression of Cxcl15 gene (also known as Il8, lungkine. http://www.ncbi.nlm.nih.gov/ 
gene/?term=Cxc115), but not the genes coding for other neutrophil chemoattractants, in the lung tissues harboring metastases from $\mathrm{T} / \mathrm{H} / \mathrm{H}-\mathrm{B} 16 \mathrm{~F} 0$ cells (Figure 3A). Interestingly, when mice were inoculated with $\mathrm{T} / \mathrm{H} / \mathrm{H}$ HepG2 or T/H/H-MCF-7 cells, 14,15-EET-treatment increased the levels of human IL 8 mRNA, but not mouse Cxcl15 mRNA in the lung tissues (Figure 3B), suggesting that 14,15 -EET induced hIL-8/mCXCL15 expression in tumor cells, but not non-cancerous cells. Indeed, 14,15-EET induced CXCL15 expression in B16F0 cells, and IL-8 expression in HepG2 and MCF-7 cells, in a dose-dependent manner at physiologically relevant concentrations (1 to $100 \mathrm{nM}$, ref. 24) (Figure 3C). The prolonged stimulation with 14,15-EET induced the continuous expression of CXCL15 (Figure 3D). CXCL15 expression in $\mathrm{B} 16 \mathrm{~F} 0$ cells was not substantially increased by other regioisomers of EET (Supplementary Figure S4A). 14,15-EET did not efficiently induce the expression of other neutrophil chemoattractants in B16F0 cells (Supplementary Figure S4B). Same results (inducing IL-8 but not other neutrophil chemoattractants) were obtained in HepG2 and MCF-7 cells (data not shown).
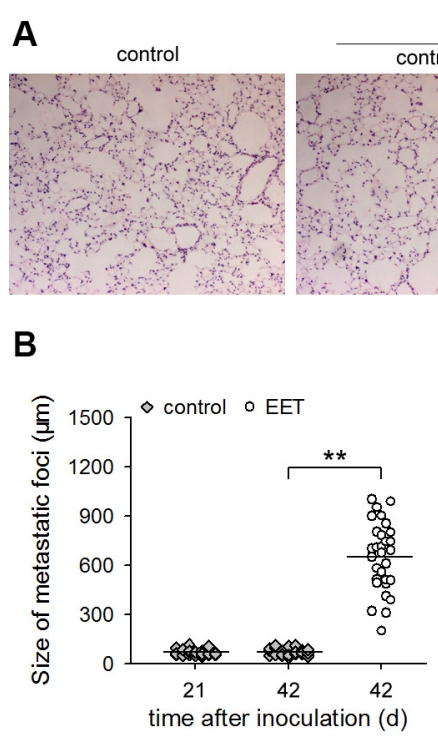

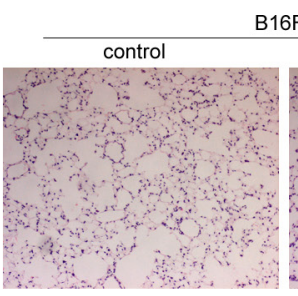

C

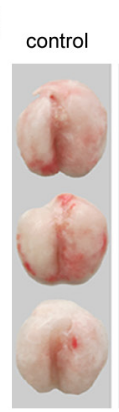

B16F0
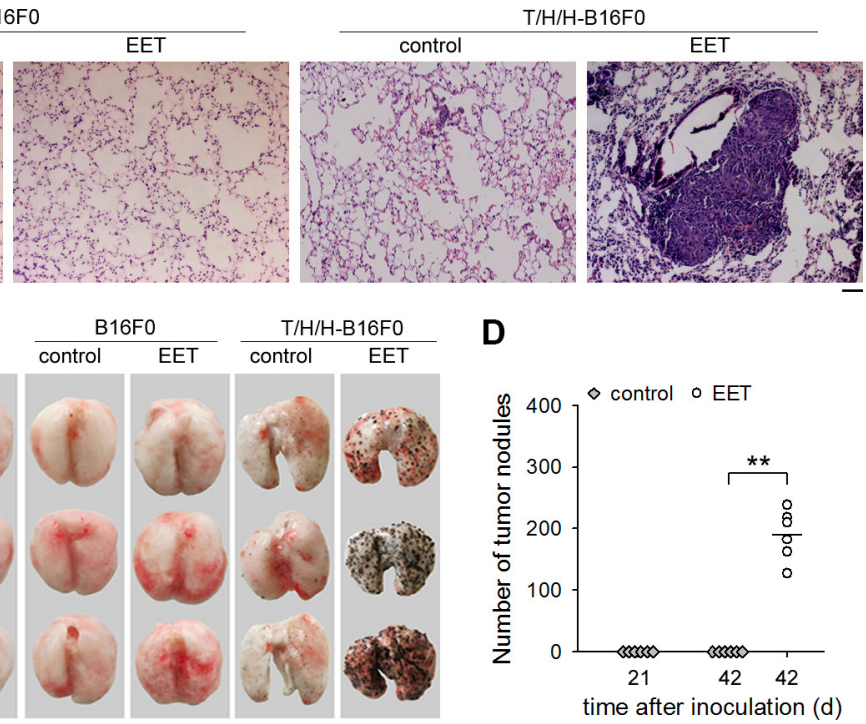

D

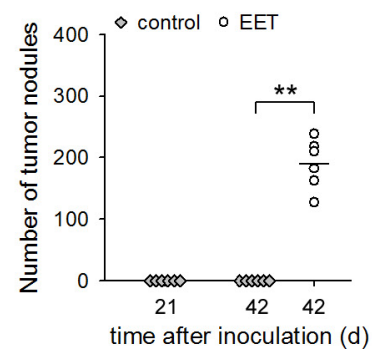

Figure 1: 14,15-EET triggers the growth of minimal dormant metastases. Mice $(n=6$ per group) were inoculated with $\mathrm{B} 16 \mathrm{~F} 0$ cells $(\mathrm{A}, \mathrm{C})$ or $\mathrm{T} / \mathrm{H} / \mathrm{H}-\mathrm{B} 16 \mathrm{~F} 0$ cells $(\mathbf{A}-\mathrm{D})$, and then were untreated or treated with 14,15-EET. The mice were sacrificed at the indicated time points $(\mathrm{B}, \mathrm{D})$ or on $\mathrm{d} 42(\mathrm{~A}, \mathrm{C})$ after inoculation. (A) Representative images of the sections of lung tissues after H\&E staining $(100 \times$ magnification, Bar, $50 \mu \mathrm{m})$. (B) Metastatic foci in tissue sections were measured as described in Methods. (C and D) Metastatic nodules on the surface of lungs (C) were counted (D). ${ }^{* *} p<0.01$.
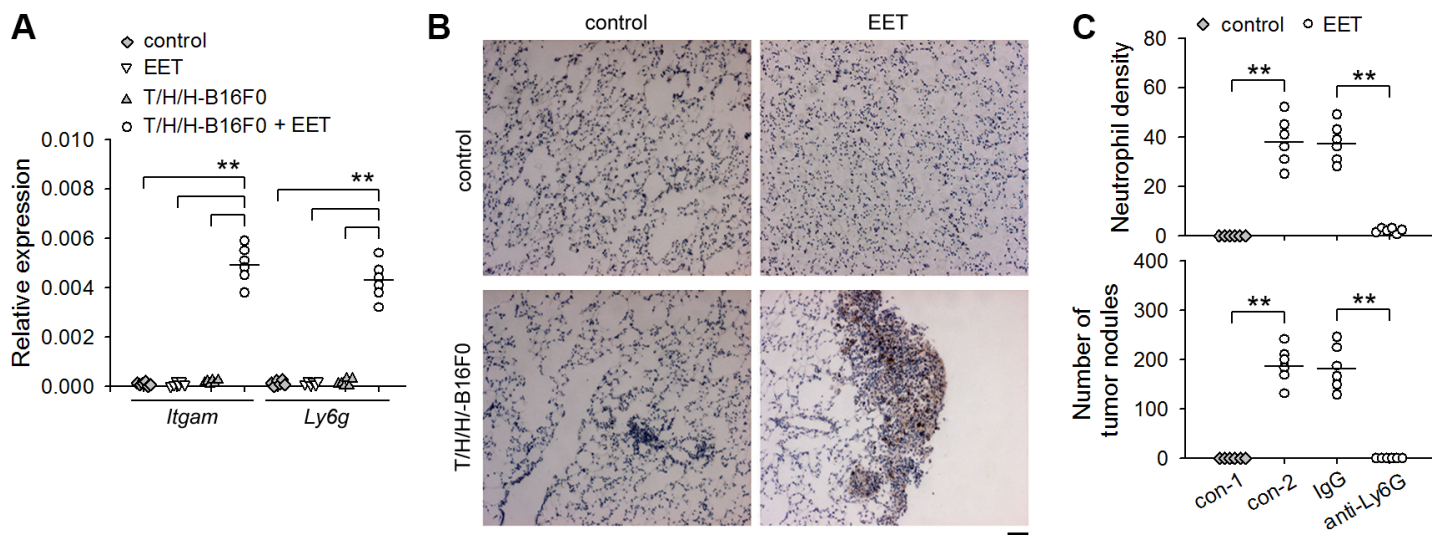

Figure 2: 14,15-EET induces neutrophilic infiltration to promote the development of metastatic lesions. Control mice (A and B) and the mice inoculated with T/H/H-B16F0 cells (A to C) were untreated or treated with 14,15-EET. The mice ( $n=6$ per group) were sacrificed on d42 after inoculation. (A) The expression of Itgam (CD11b) and Ly6g (Ly6G) genes in lung tissues was detected by real-time RT-PCR. (B) The sections of lung tissues were subjected to immunohistochemical staining for identifying the infiltration of neutrophils. Bar, $50 \mu \mathrm{m}$. (C) Anti-Ly6G antibody was used to deplete neutrophils in vivo when the mice were treated with 14,15-EET. Neutrophil density in lung tissue sections was determined after immunohistochemical staining as described in Methods (upper). Metastatic nodules on the surface of lungs were counted (lower). ${ }^{* *} p<0.01$. 
To clarify the crucial role of tumor cell-derived hIL-8/mCXCL15, we used shRNA to inhibit the upregulation of hIL-8/mCXCL15 (Supplementary Figure S4C). When the up-regulation of CXCL15 (B16F0 cells) and IL-8 (HepG2 cells) was inhibited, 14,15-EET failed to promote the neutrophilic infiltration and the development of micrometastases (Figure 3E and Supplementary Figure S4D), suggesting that 14,15-EET indeed promoted neutrophilic infiltration in metastatic lesions by up-regulating neutrophil chemoattractant expression in tumor cells.

\section{4,15-EET promotes hIL-8/mCXCL15 expression in tumor cells by activating STAT3 and JNK pathways}

In different types of cells, IL-8 expression could potentially be induced or promoted by different signaling pathways, including NF-кB, ERK, p38 MAPK, JNK, and STAT3 pathways [25-28]. To identify the signaling pathway(s) required for 14,15-EET to induce hIL-8/ mCXCL15 expression in tumor cells, we detected the activation of these pathways by 14,15 -EET. The results showed that 14,15-EET could activate STAT3 and three MAPK pathways, but not NF- $\kappa$ B pathway, in B16F0 (Figure 4A) and HepG2 cells (Supplementary Figure S5A). We then stimulated these cells with 14,15-EET in presence of STAT3 inhibitor VIII, PD98059 (inhibitor of ERK pathway), SB203580 (p38 MAPK inhibitor), SP600125 (JNK inhibitor), and QNZ (NF- $\mathrm{B}$ inhibitor). hIL-8/ mCXCL15 expression was not significantly influenced by the inhibitors of ERK, p38 MAPK, and NF-кB pathways. Inhibiting JNK pathway suppressed both early up-regulation (first $48 \mathrm{~h}$ ) and the continuous expression of CXCL15 in B16F0 cells (Figure 4B, 4C) and IL-8 in HepG2 cells (Supplementary Figure S5B, S5C). Consistently, the prolonged treatment with 14,15-EET

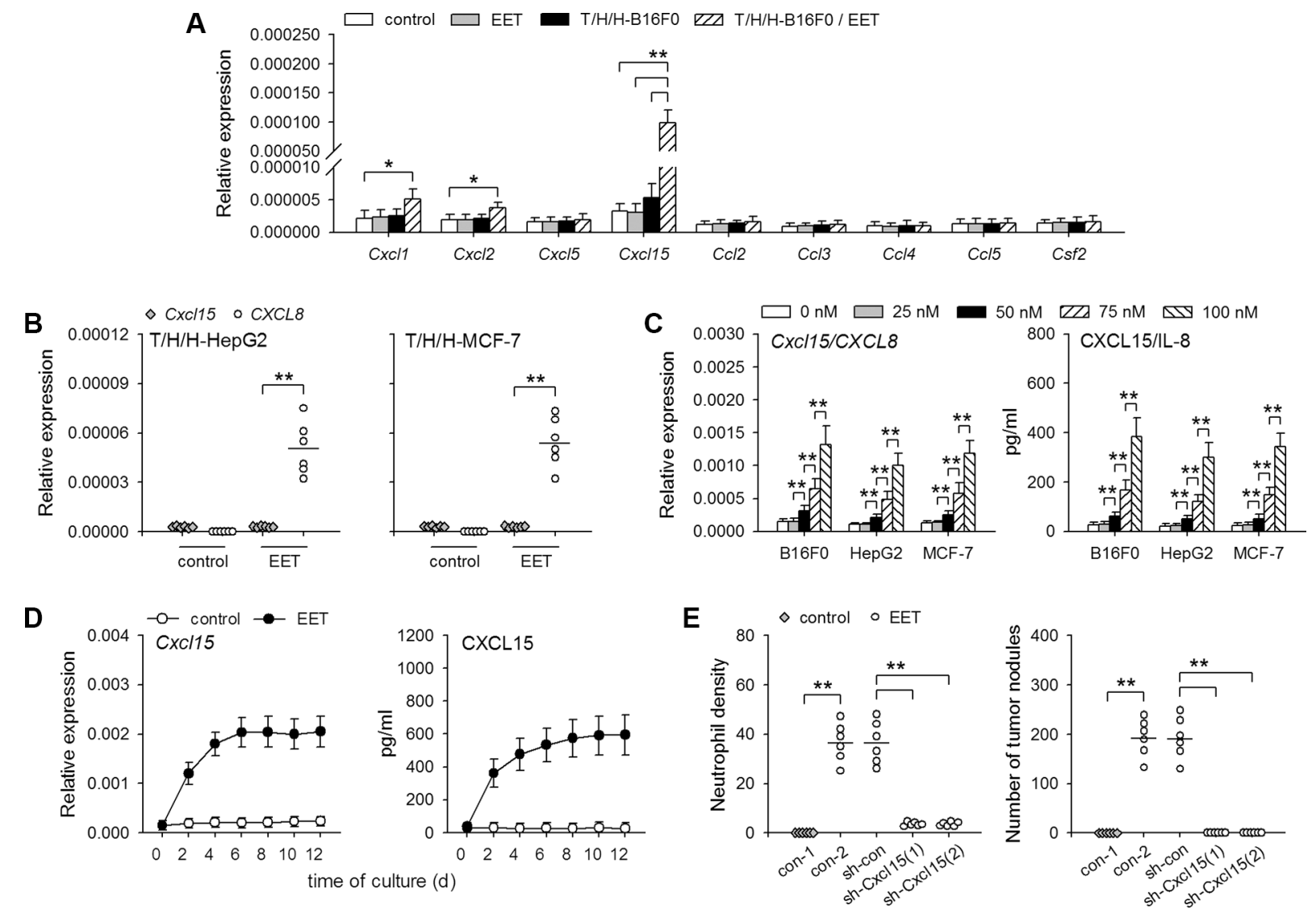

Figure 3: 14,15-EET induces hIL-8/mCXCL15 expression in tumor cells. (A) Control mice and the mice inoculated with $\mathrm{T} / \mathrm{H} / \mathrm{H}-\mathrm{B} 16 \mathrm{~F} 0$ cells were untreated or treated with 14,15-EET. The mRNA levels of the indicated mouse genes in lung tissues were detected by real-time RT-PCR on d42 after inoculation ( $n=6$ per group). (B) Mice inoculated with T/H/H-HepG2 cells or T/H/H-MCF-7 cells were untreated or treated with 14,15-EET. On d42 after inoculation, the mRNAs of mouse Cxcl15 gene and human CXCL8 (IL8) gene in lung tissues were detected by real-time RT-PCR, and both were quantified against mouse Gapdh mRNA ( $n=6$ per group). (C) Tumor cells were untreated or treated with 14,15-EET for $48 \mathrm{~h}$ at the indicated concentrations. The expression of Cxcl15/CXCL8 gene was detected by real-time RT-PCR and ELISA ( $n=6$ per group). (D) B16F0 cells were untreated or treated with 14,15-EET. The expression of $C x c l 15$ gene was detected by real-time RT-PCR and ELISA at the indicated time points. (E) B16F0 cells, untransfected or transfected with the indicated vectors, were inoculated to mice after T/H/H-treatment. The mice, untreated or treated with 14,15-EET, were sacrificed on $\mathrm{d} 42$ after inoculation. Neutrophil density in lung tissue sections was determined after immunohistochemical staining (left). Metastatic nodules on the surface of lungs were counted (right). $* * p<0.01$. 
induced the sustained and gradually enhanced activation of JNK pathway (Figure 4D and Supplementary Figure S5D). Differently, 14,15-EET did not induce the gradually enhanced STAT3 activation. The later inhibition of STAT3 pathway had less effect on hIL-8/mCXCL15 expression. However, the early inhibition of STAT3 pathway significantly suppressed both early up-regulation and the continuous expression of hIL-8/mCXCL15. These results suggest that the activation of STAT3 pathway might not only induce early up-regulation of hIL-8/mCXCL15 in tumor cells, but also augment the later effect of JNK pathway.

\section{miR-155 is required for $14,15-E E T$ to maintain the continuous expression of hIL-8/mCXCL15}

To further clarify the mechanism underlying the continuous expression of hIL-8/mCXCL15 induced by 14,15 -EET, we investigated whether 14,15-EET might down-regulate or up-regulate the expression of microRNAs. In different types of cells, several microRNAs have been found to influence IL- 8 production. miR-93, miR-106b, and miR-203 directly bind IL8 mRNA to suppress translation $[29,30]$. miR-16, miR-31,
miR-33a, miR-146a, miR-155, and miR-301a can suppress or promote IL-8 expression and secretion by inhibiting the expression of different proteins [31-36]. Among them, miR-155 was substantially up-regulated by $14,15-$ EET (Figure 5A). Interestingly, the prolonged treatment with 14,15-EET also induced the continuous expression of miR-155 (Figure 5B and Supplementary Figure S6A). 14,15-EET up-regulated the expression of miR-155 through STAT3 and JNK pathways (Figure 5C and 5D, and Supplementary Figure S6B, S6C). The early inhibition of STAT3 pathway significantly suppressed both early upregulation (first $48 \mathrm{~h}$ ) and the continuous expression of miR-155, whereas the later inhibition of STAT3 pathway had less effect on the continuous expression of miR-155. Inhibiting JNK pathway mainly suppressed the continuous expression of miR-155.

miR-155 does not directly bind IL- 8 mRNA, but it can promote the activation of JNK pathway by downregulating the expression of SHIP1 and DET1 [35, 37]. Consistent with the expression pattern of miR-155, the expression of SHIP1 and DET1 was gradually downregulated, and the expression and activation of c-Jun was gradually augmented in B16F0 and HepG2 cells after prolonged treatment with 14,15-EET (Figure 5E
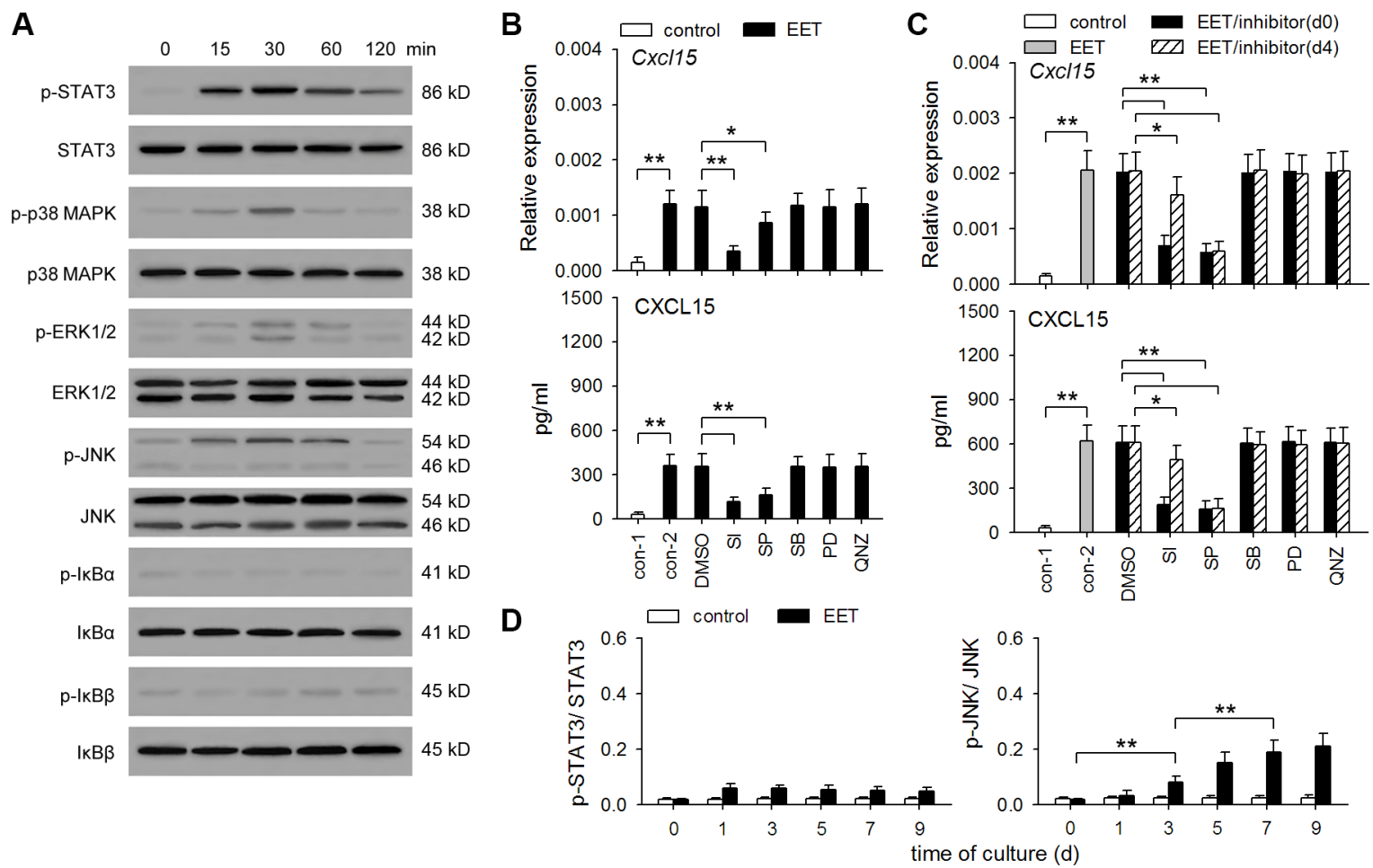

Figure 4: 14,15-EET activates STAT3 and JNK pathways to up-regulate CXCL15 expression. (A) B16F0 cells were stimulated with 14,15-EET (100 nM). The phosphorylation of STAT3, p38 MAPK, ERK, JNK, IкB $\alpha$, IкB $\beta$ was detected by Western blot at the indicated time points. Data are representative of three independent experiments. (B and C) B16F0 cells were untreated or treated with 14,15-EET for $48 \mathrm{~h}$ (B) or 10 days (C) in absence or presence of STAT3 inhibitor VIII (SI, $50 \mu \mathrm{M})$, SP600125 (10 $\mu \mathrm{M})$, SB203580 (10 $\mu \mathrm{M})$, PD98059 $(10 \mu \mathrm{M})$, and QNZ $(40 \mathrm{nM})$. The inhibitors were added on $\mathrm{d} 0$ (B and C) or $\mathrm{d} 4(\mathrm{C})$ respectively. The expression of $C x c l 15 \mathrm{gene}$ was detected by real-time RT-PCR and ELISA. (D) B16F0 cells were unstimulated or stimulated with 14,15-EET. The ratios of phospho-STAT3 to STAT3 (p-STAT3/STAT3) or phospho-JNK to JNK (p-JNK/JNK) at the indicated time points were calculated after densitometric analysis of Western blots. Data are pooled from three independent experiments with a total of six samples in each group. ${ }^{*} p<0.05$, $* * p<0.01$. 
and Supplementary Figure S6D). When these cells were stimulated with 14,15 -EET in presence of miR-155 inhibitor, 14,15-EET did not suppress SHIP1 and DET1 expression (Supplementary Figure S6E), failed to induce the enhanced and sustained activation of JNK and c-Jun (Figure 5F and Supplementary Figure S6F), and was unable to maintain the continuous expression of CXCL15 (Figure 5G) and IL-8 (Supplementary Figure S6G). Taken together, these results suggest that 14,15-EET could up-regulate the expression of miR-155 to maintain the enhanced and sustained activation of JNK pathway, thus maintaining the continuous expression of hIL-8/ mCXCL15.

\section{Neutrophil function is converted by $14,15-E E T-$ treatment in vivo}

The above results indicated that 14,15-EET-induced neutrophilic infiltration could promote the growth of dormant metastases. We then further investigated the function of neutrophils in a co-inoculation test (Figure 6A) and the metastatic model using $\mathrm{T} / \mathrm{H} / \mathrm{H}-\mathrm{B} 16 \mathrm{~F} 0$ or metastatic cell line B16F1 (Supplementary Figure S7A-S7E). Simply harboring dormant metastases could not induce the conversion of neutrophil function from tumor-suppressing to tumor promoting (Figure 6A). The conversion was induced by treating mice with 14,15-EET (Figure 6A,
A

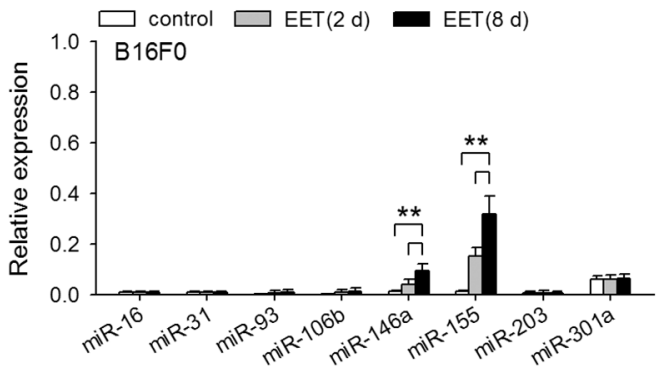

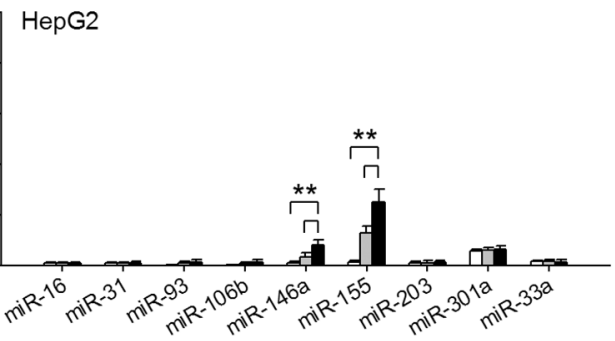

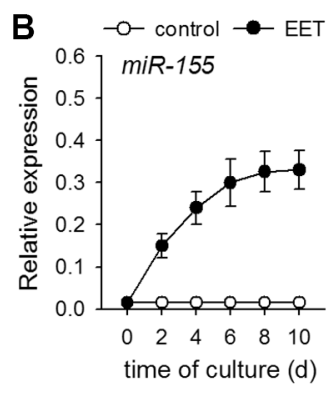

E

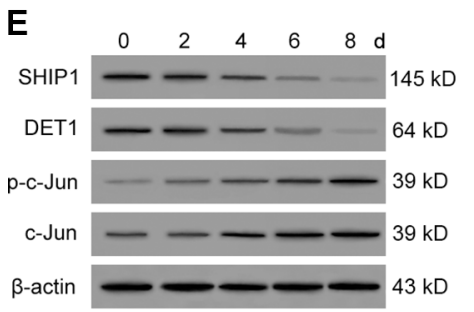

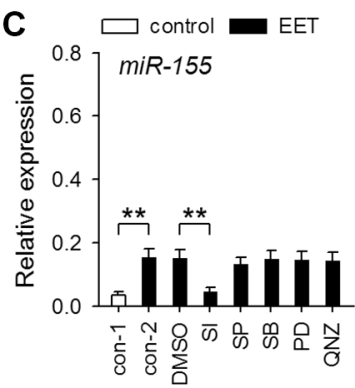

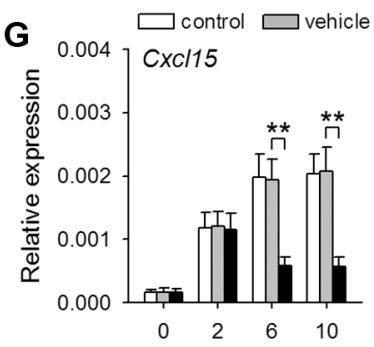

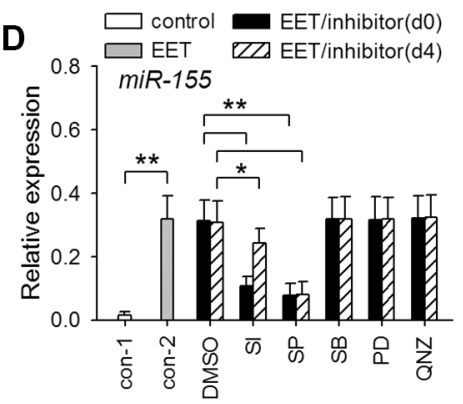

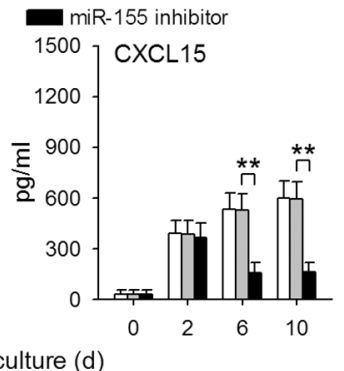

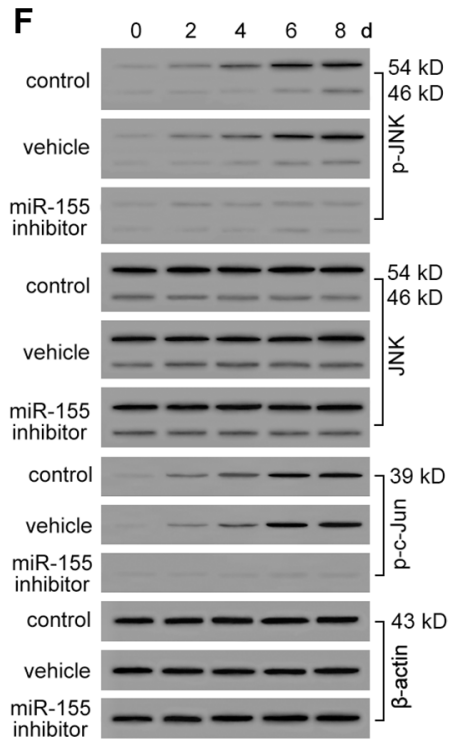

Figure 5: 14,15-EET up-regulates miR-155 expression to maintain the sustained activation of JNK pathway. (A) B16F0 cells and HepG2 cells were untreated or treated with 14,15-EET (100 nM) for 2 days or 8 days. The expression of the indicated microRNAs was detected by real-time RT-PCR ( $n=6$ per group). (B to E) B16F0 cells were untreated or treated with 14,15-EET. (B) The expression of miR-155 was detected by real-time RT-PCR at the indicated time points. (C and D) The cells were cultured for $48 \mathrm{~h}(\mathrm{C})$ or $10 \mathrm{days}(\mathrm{D})$ in absence or presence of STAT3 inhibitor VIII (SI, $50 \mu \mathrm{M})$, SP600125 (10 $\mu \mathrm{M})$, SB203580 (10 $\mu \mathrm{M})$, PD98059 (10 $\mu \mathrm{M})$, and QNZ (40 nM). The inhibitors were added on $\mathrm{d} 0$ (C and D) or $\mathrm{d} 4$ (D) respectively. The expression of miR-155 was detected by real-time RT-PCR. (E) The expression of SHIP1, DET1, c-Jun, and the phosphorylation of c-Jun, were detected by Western blot at the indicated time points. (F and $\mathbf{G})$ B16F0 cells were treated with 14,15-EET in absence or presence of miR-155 inhibitor (50 nM) or vehicle. The phosphorylation of JNK and c-Jun was detected by Western blot at the indicated time points (F). The expression of Cxcl15 gene was detected by real-time RT-PCR and ELISA at the indicated time points (G). Data are representative of three independent experiments (E, F). ${ }^{*} p<0.05,{ }^{* *} p<0.01$. 
Supplementary Figure S7A-S7D), or increasing in vivo production of EETs with the inhibitor of soluble epoxide hydrolase (sEH, the enzyme that metabolizes 14,15-EET) (Supplementary Figure S7E). Consistently, the neutrophils isolated from 14,15-EET-treated mice, but not the mice simply harboring dormant metastases, produced more MMP-9 (Figure 6B) and expressed much less TRAIL (Figure 6C), which is in favor of angiogenesis.

We then analyzed whether 14,15-EET may influence the activation of STAT3 in neutrophils, since the enhanced activation of STAT3 results in the conversion of neutrophil function [12]. The results showed that both the expression and activation of STAT3 in neutrophils were increased by 14,15-EET-treatment in vivo, but not by simply harboring dormant metastases (Figure 6D and Supplementary Figure S7F). Intriguingly, the treatment with 14,15-EET, but not other regioisomers, substantially increased the serum levels of G-CSF and IL-6 (Figure 6E, and Supplementary Figure S7G). Moreover, 14,15-EET could cooperate with G-CSF/IL-6 to enhance the activation of STAT3 in neutrophils (Figure 6F), increasing the expression of Mmp 9 gene and decreasing the expression of Trail gene (Supplementary Figure S7H). Taken together, these results suggest that 14,15 -EET could induce the enhanced activation of STAT3 in neutrophils.

\section{Neutrophil-derived MMP-9 is crucial for 14,15- EET to induce the growth of dormant metastases}

We next investigated whether neutrophil-derived MMP-9 is crucial for 14,15-EET to trigger the growth of dormant metastases. 14,15-EET dramatically increased the level of $M m p 9$ mRNA in the lung tissues harboring dormant metastases from $\mathrm{T} / \mathrm{H} / \mathrm{H}-\mathrm{B} 16 \mathrm{~F} 0$ cells, but not in
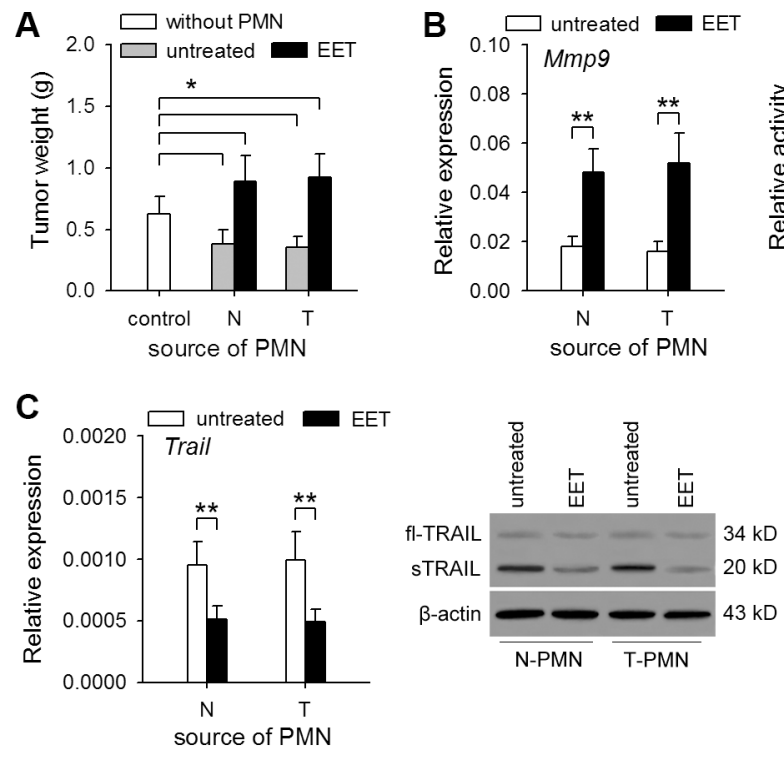

E

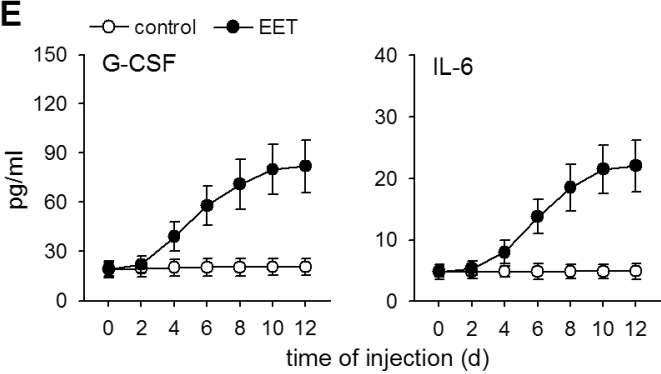

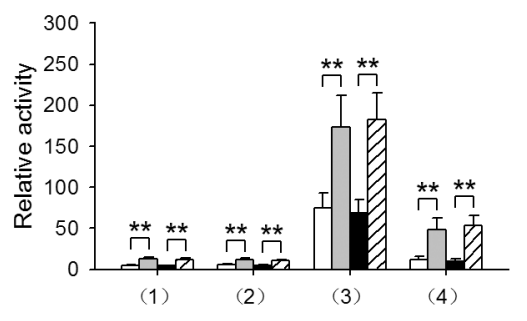
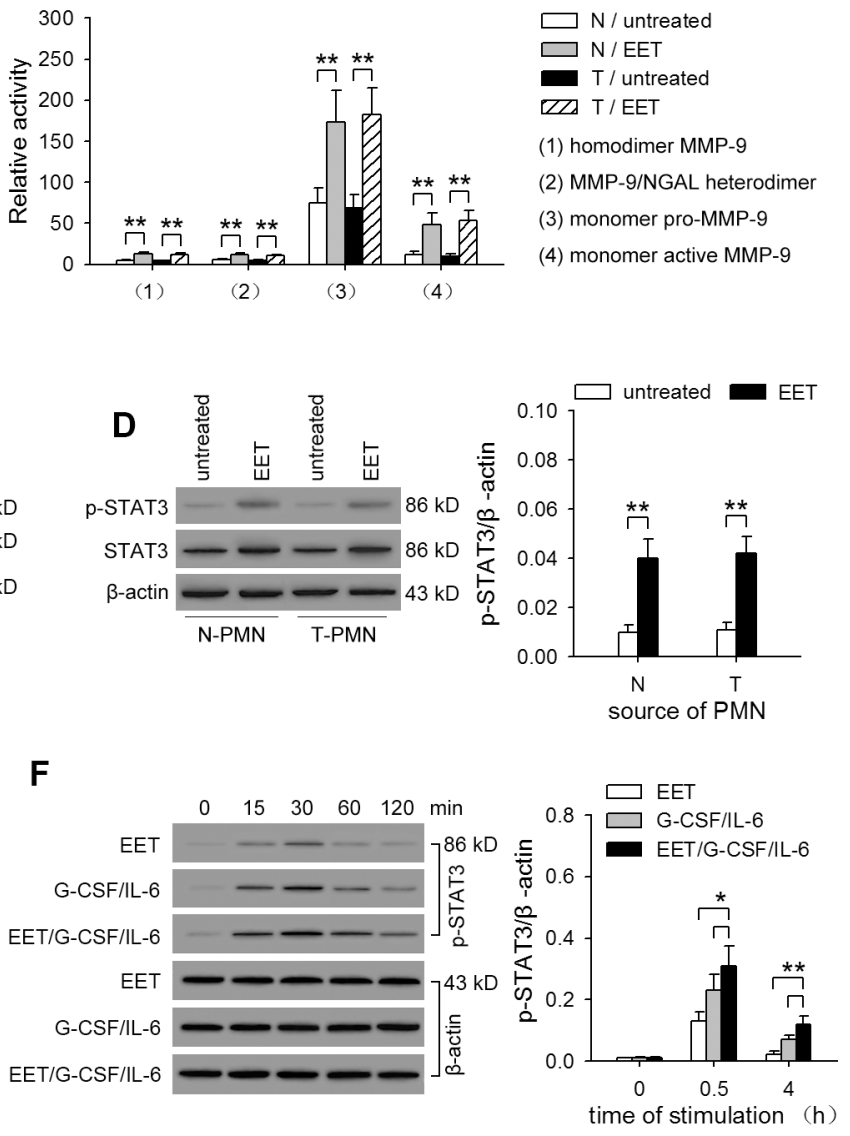

Figure 6: 14,15-EET induces tumor-promoting the function of neutrophils. (A to D) Control mice (N) and the mice inoculated with T/H/H-B16F0 cells (T) were untreated or treated with 14,15-EET. On d42 after inoculation, neutrophils were isolated from the peritoneal cavity of mice after recruitment as described in Methods. (A) Neutrophils were used for co-inoculation test as described in Methods. (B) The expression and release of MMP-9 by neutrophils were detected by real-time RT-PCR and zymography. (C) The expression of TRAIL in neutrophils was detected by real-time RT-PCR and Western blot. (D) STAT3 and phospho-STAT3 in neutrophils was detected by Western blot (left). The relative level of phospho-STAT3 to $\beta$-actin was calculated after densitometric analysis of Western blots (right). (E) The levels of serum G-CSF and IL-6 in naive mice and 14,15-EET-treated mice were detected by ELISA. (F) Neutrophils were isolated from bone marrow of naive mice, and stimulated with 14,15-EET (100 nM) and/or G-CSF/IL-6 (50 ng/ml of each). The phospho-STAT3 (p-STAT3) was analyzed at the indicated time points by Western blot (left). The relative level of p-STAT3 to $\beta$-actin was calculated after densitometric analysis of Western blots (right). Data are representative of three independent experiments (C, D, F, Western blots), or pooled from three independent experiments with a total of six samples in each group (D right, F right). ${ }^{*} p<0.05,{ }^{*} p<0.01$. 
control lung tissues (Figure 7A). Interestingly, when mice were inoculated with $\mathrm{T} / \mathrm{H} / \mathrm{H}-\mathrm{HepG} 2$ or $\mathrm{T} / \mathrm{H} / \mathrm{H}-\mathrm{MCF}-7$ cells, 14,15-EET mainly increased the levels of mouse Mmp 9 mRNA in lung tissues, but only slightly increased the levels of human MMP9 mRNA (Supplementary Figure S8A). If neutrophils were depleted in vivo, 14,15-EET could not efficiently increase $M m p 9$ mRNA in the lung tissues harboring metastases from B16F0, HepG2, and MCF-7 cells (Figure 7B, and Supplementary Figure S8B). These results suggested that neutrophils were the main source of MMP-9 in the lung tissues after 14,15-EETtreatment.

After inoculation with $\mathrm{T} / \mathrm{H} / \mathrm{H}-\mathrm{B} 16 \mathrm{~F} 0$ cells and 14,15-EET-treatment, the angiogenesis in metastatic lesions was observed in control mice, but not neutrophil-depleted mice (Figure 7C) or MMP-9-/- mice (Supplementary Figure S8C), similar to the effect of blocking VEGF in vivo (Supplementary Figure S8D). 14,15-EET could promote the proliferation of tumor cells (Supplementary Figure S8E and ref. 16). However, in neutrophil-depleted mice (Figure 7D, 7E, and Supplementary Figure S8F), MMP-9 ${ }^{-/-}$mice (Supplementary Figure S8G), and VEGF-blocking mice (Supplementary Figure S8H), 14,15-EET only slightly increased the size of microscopic metastatic foci, but did not result in the formation of macroscopic metastases. Therefore, MMP-9 from neutrophils is crucial for 14,15EET to promote the angiogenesis in metastatic lesions and the development of metastatic lesions.

\section{DISCUSSION}

Infiltrating neutrophils could promote tumor angiogenesis and tumor progression [6, 12]. Here,
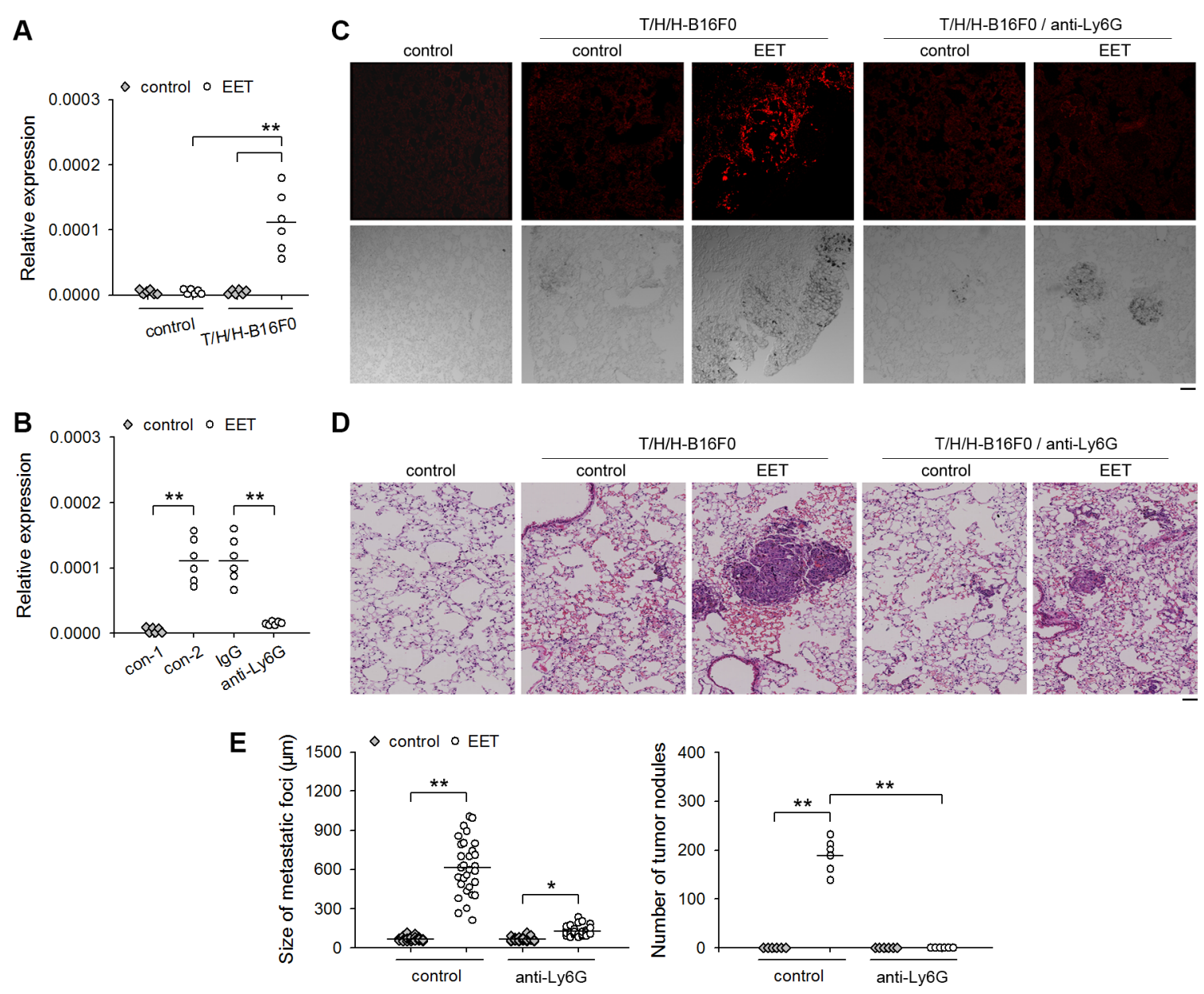

Figure 7: Neutrophils are the main source of MMP-9 in 14,15-EET-induced development of metastatic lesions. The mice inoculated with T/H/H-B16F0 cells were untreated or treated with 14,15-EET as described in Methods. Neutrophils were not depleted (A) or depleted in vivo with anti-Ly6G antibody (B to E) when the mice were treated with 14,15-EET. The mice ( $n=6$ per group) were sacrificed on $\mathrm{d} 42$ after inoculation. (A and B) The expression of Mmp9 gene in lung tissues was detected by real-time RT-PCR. The mice without inoculation were also used as control in A. (C and D) Lung tissue sections were stained for CD34 to identify microvessels (C), or subjected to H\&E staining (D). Representative photographs are shown. Bar, $50 \mu \mathrm{m}$. The mice without inoculation were also used as control in C and D. (E) Metastatic foci in tissue sections (H\&E staining) were measured (left). Metastatic nodules on the surface of lungs were counted (right). ${ }^{*} p<0.05,{ }^{* *} p<0.01$. 
our results suggest that the inefficient recruitment of neutrophils might be an important reason that the dormancy of micrometastases could be maintained. Non-metastatic tumor cells may acquire invasive capacity in presence of TGF- $\beta 1$ and neutrophil-derived $\mathrm{H}_{2} \mathrm{O}_{2}$ and $\mathrm{HOCl}$, but the cells could only form dormant micrometastases after extravasation as shown in our present and previous studies [19]. Intriguingly, our data in this study showed that 14,15 -EET could trigger the growth of dormant micrometastases by inducing neutrophil infiltration in metastatic lesions and the conversion of neutrophil function, which might be a driving force for tumor recurrence based on dormant metastases.

EETs have been known to exert antiinflammatory effects in vivo [14, 15]. Intriguingly, however, 14,15EET could induce neutrophilic infiltration in metastatic lesions by activating STAT3 and JNK pathways to induce the expression of IL-8 and CXCL15 in tumor cells. Interestingly, 14,15-EET increased IL-8 expression in human tumor cells and CXCL15 expression in mouse tumor cells by activating same signaling pathways. JNK pathway can promote both expression and secretion of IL-8 [25, 38]. 14,15-EET could induce the enhanced and sustained activation of JNK pathway by increasing miR-155 expression. 14,15-EET was less efficient in inducing the transient activation of JNK pathway in tumor cells due to the inhibitory effect of SHIP1 and DET1. Therefore, although JNK pathway could promote the expression of miR-155 as shown by our data and others [39], the early up-regulation of miR-155 was mainly induced by 14,15-EET-activated STAT3. When miR-155 was up-regulated, JNK/c-Jun pathway was easier to be activated due to the down-regulation of SHIP1 and DET1 expression by miR-155. Then, the enhanced activation of JNK pathway was more efficient in maintaining the expression of miR-155, thus forming a positive feedback loop to maintain the enhanced and sustained activation of JNK pathway, and the continuous production of hIL-8/ mCXCL15 that is important for the continuous recruitment of neutrophils and the development of metastatic lesions.

Although neutrophils have the potential to inhibit metastatic seeding in the premetastatic lungs [40], 14,15EET could induce the conversion of neutrophil function in vivo. The enhanced activation of STAT3 in neutrophils can promote the expression and release of MMP-9, and reduce the expression of TRAIL and the release of NE [12]. The direct effect of 14,15-EET alone on neutrophils was not strong, since the activation of STAT3 in neutrophils is not as efficient as that in other types of cells [12]. The increased STAT3 expression and the costimulation of different factors, such as G-CSF/IL-6, are required to enhance STAT3 activation in neutrophils [12]. IL- 6 can down-regulate IFN- $\beta$ expression in bone-marrow to increase STAT3 expression in neutrophils [12]. G-CSF and IL- 6 can potentially be produced by different cells in the setting of chronic inflammation, including endothelial cells, fibroblasts, monocytes, and other cells [41, 42]. 14,15-EET might stimulate some of these cells to increase the production of G-CSF and IL-6 in vivo. Moreover, 14,15-EET can cooperate with G-CSF/IL-6 to more efficiently induce the enhanced and sustained activation of STAT3, thus inducing the conversion of neutrophil function from tumor-suppressing to tumor-promoting.

All of four regioisomeric EETs have the potential to promote angiogenesis by directly stimulating endothelial cells [43]. However, only 14,15-EET could efficiently trigger the growth of dormant micrometastases as shown by our data, since only 14,15-EET could induce neutrophilic infiltration and convert neutrophil function. Neutrophils produce MMP-9 but not tissue inhibitors of metalloproteinases (TIMPs). Therefore, neutrophils release TIMP-free MMP-9 [7, 44], which is rapidly and freely available to promote angiogenesis by remodeling extracellular matrix and releasing such potent angiogenic factors as VEGF and FGF2 that are usually sequestered in an inactivated form to the ECM [7]. In contrast to neutrophils, other MMP-9-producing cells produce TIMPs, and therefore release inactive MMP-9/TIMP complexes [7, 44]. 14,15-EET could promote MMP9 expression in tumor cells, but did not increase the production of active MMP-9 (our unpublished data). On the other hand, our data showed that neutrophils were the main source of MMP-9 in the lung tissues harboring metastases after 14,15-EET treatment. In the absence of neutrophils, 14,15-EET failed to efficiently increase $\mathrm{Mmp} 9$ gene expression in the lung tissues harboring metastases. Therefore, inducing neutrophilic infiltration in metastatic lesions is crucial for 14,15 -EET to activate angiogenesis and trigger the growth of dormant metastases. Thereafter, the other three regioisomeric EETs may promote the angiogenesis by stimulating endothelial cells.

14,15-EET promotes the growth of dormant micrometastases in a dose-dependent manner. Our data might have the implication that the increase of 14,15-EET in vivo might increase the risk of tumor recurrence based on the dormant micrometastases. EETs can be increased by factors that promote P450 expression and/or activity, or by chemicals and nutritional factors [45]. Therefore, controlling the EET levels in patients is of importance to prevent the growth of dormant micrometastases. In addition to 14,15-EET, the growth of dormant micrometastases might also be triggered by other factors through similar mechanisms. For example, infection could induce the production of G-CSF/IL-6 and $\mathrm{PGE}_{2}$ [46]. PGE 2 could induce the expression of CXCL1 in tumor cells [47]. Therefore, infection-induced chronic inflammation might also promote the recruitment of tumor-promoting neutrophils to metastatic lesions. Given that there are different chemoattractants, and that protracted depletion of neutrophils is clinically untenable, exploring the approach for augmenting tumor-suppressing function of neutrophils might be very important for designing the therapeutic 
strategy for preventing the recurrence of tumor based on metastases.

\section{MATERIALS AND METHODS}

\section{Cells and treatment}

C57BL/6 background B16F0 and B16F1 melanoma cells, human hepatocellular carcinoma HepG2 cells, and human breast cancer MCF-7 cells were purchased from China Center for Type Culture Collection (CCTCC, Wuhan, China) and cultured according to their guidelines. The cell lines were authenticated at China Center for Type Culture Collection (Wuhan, China) in June 2014, using short tandem repeat (STR) DNA profiling (ABI 31300xl Genetic Analyzer; Life Technologies). To induce the invasive capacity of non-metastatic tumor cells (B16F0, HepG2, MCF-7), the cells were cultured in presence of $\mathrm{T} / \mathrm{H} / \mathrm{H}$ (TGF- $\beta 1,5 \mathrm{ng} / \mathrm{ml}, \mathrm{H}_{2} \mathrm{O}_{2}, 100 \mu \mathrm{M}, \mathrm{HOCl}, 50 \mu \mathrm{M}$ ) for 10 days [19]. For the convenience of description, $\mathrm{T} / \mathrm{H} / \mathrm{H}$-pretreated cells were mentioned as $\mathrm{T} / \mathrm{H} / \mathrm{H}-\mathrm{B} 16 \mathrm{~F} 0$, $\mathrm{T} / \mathrm{H} / \mathrm{H}-\mathrm{HepG} 2$, and T/H/H-MCF-7 cells, respectively.

\section{Animals}

C57BL/6 mice (6-8 weeks old) were purchased from the Center of Medical Experimental Animals of Hubei Province (Wuhan, China). B6.FVB $(\mathrm{Cg})-M_{m p} 9^{t m 1 T v u} / \mathrm{J}$ mice (MMP-9-/) were kindly provided by professor HongLiang Li (Cardiovascular Research Institute of Wuhan University). Athymic nude (nu/nu) mice (4-5 weeks old) were purchased from Beijing HFK Bio-Technology Co. LTD. (Beijing, China). The mice were maintained in the accredited animal facility of Tongji Medical College, and animal care was in accordance with institutional guidelines. All animal experiments were approved by the Committee on the Ethics of Animal Experiments of Tongji Medical College (Permit Number: 2012-S395).

\section{Metastasis model and EET-treatment}

To establish the model of minimal dormant metastases, C57BL/6 mice or $\mathrm{MMP}-9^{-/}$mice were inoculated with $\mathrm{B} 16 \mathrm{~F} 0$ cells $\left(5 \times 10^{5}\right.$ cells/mouse $)$, or nude mice were inoculated with HepG2 cells $\left(4 \times 10^{6}\right.$ cells/mouse) or MCF-7 cells $\left(2 \times 10^{6}\right.$ cells/mouse $)$. These non-metastatic tumor cells acquired invasive capacity after pretreatment with $\mathrm{T} / \mathrm{H} / \mathrm{H}$, and were able to extravasate after injection via tail vein (Supplementary Figure S1A and refs. 19, 20). 3 weeks after inoculation, only microscopic metastatic lesions were observed in lung tissues, and no net size increase in metastatic lesion occurred thereafter (Supplementary Figure S1B and refs. 19, 20). The dormancy of micrometastases was also shown by the reduced accumulation of $\mathrm{Ki}-67$, a proliferation marker, in tumor cells (Supplementary Figure S1C and ref. 20). When indicated, the mice received the i.v. injection of 14,15-EET $(30 \mu \mathrm{g} / \mathrm{kg})$ and/or 14,15-EEZE $(30 \mu \mathrm{g} / \mathrm{kg})$ or 14,15 -DHET $(30 \mu \mathrm{g} / \mathrm{kg})$, once every two days, from d22 to d40 after inoculation (Supplementary Figure S1D). The mice were sacrificed on the indicated time points. In B16F0-model, the black nodules, but not faint brown spots, on the surface of lung were counted as visible metastatic nodules. In HepG2- and MCF-7-model, the lungs of mice were fixed in Bouin's solution (Sigma Aldrich), displaying metastatic nodules on the surface of lung as darker color nodules.

\section{In vivo depletion of neutrophils}

To deplete neutrophils, anti-Ly6G antibody was used [13]. Starting from d3 after first injection of 14,15-EET, the mice received i.p. injection of anti-Ly6G antibody at a dose of $300 \mu \mathrm{g}$ in $500 \mu \mathrm{l} \mathrm{PBS}$ [48], once every three days for six times. The depletion of neutrophils was identified by flow cytometric analysis (Supplementary Figure S3B).

\section{Cell transfection}

To inhibit the up-regulation of CXCL15 expression in B16F0 cells, the cells were transduced with sh-Cxcl15(1) and sh-Cxcl15(2) lentiviral particles (GeneChem, Shanghai, China) to express Cxcl15 shRNAs, binding 5'-CCAATTACTAACAGGTTCCTA-3' and 5'-CCTGAGAACAAGAGAATATTT-3', respectively. To suppress the up-regulation of IL-8 expression in HepG2 cells, the cells were transduced with sh-IL8(1) and shIL8(2) lentiviral particles (GeneChem) to express IL-8 shRNAs, binding 5'-CAAGGAGTGCTAAAGAACT TA-3' and 5'-GCTCTGTGTGAAGGTGCAGTT-3', respectively. sh-con, not binding any known mRNA, were used as control. After selection with puromycin, the cells were used for further experiments. To suppress the function of miR-155, the cells were cultured in the presence of miR-155 inhibitor/HiPerFect Transfection Reagent (Qiagen, Valencia, CA) according to the manufacturer's instructions.

\section{Recruitment of neutrophils to peritoneal cavity}

To recruit neutrophils to peritoneal cavity, CXCL15expressing hepatocytes were injected to peritoneal cavity of mice $\left(3 \times 10^{5}\right.$ per mouse). $12 \mathrm{~h}$ later, the peritoneal cells were harvested for the isolation of neutrophils. To acquire CXCL15-expressing hepatocytes, mice received i.v. injection of pCxcl15 plasmid (200 $\mu \mathrm{g}$ per mouse). $12 \mathrm{~h}$ later, hepatocytes were prepared from liver by two-step collagenase perfusion technique [12]. 


\section{Isolation of neutrophils}

Murine neutrophils were isolated from bone marrow cells or peritoneal cells as described previously [12]. Briefly, the cells were washed once in HBSS, layered over a Percoll gradient $(54 \% / 64 \% / 72 \%$ for bone marrow cells and $54 \% / 64 \% / 80 \%$ for peritoneal cells), and centrifuged at $1060 \times g$ for $30 \mathrm{~min}$. The dense band at $64 \% / 72 \%$ or $64 \% / 80 \%$ interface was collected as neutrophil fraction. The isolated cells were $>90 \%$ neutrophils as assessed by flow cytometric analysis and Giemsa-Wright stain (Supplementary Figure S9).

\section{Co-inoculation test}

Mice were inoculated intramuscularly in the right hind thigh with $3 \times 10^{5} \mathrm{~B} 16 \mathrm{~F} 0$ cells, mixed with $1 \times 10^{6}$ neutrophils. Tumors were dissected and weighed on $\mathrm{d} 12$ after inoculation.

\section{Reagents and other methods}

Reagents are shown in Supplementary Material. Other methods were performed using standard protocols, including assay of tumor cell arrest in lung and extravasation, histology, ELISA analysis, Western blot assay, MMP-9 assay, immunofluorescence, analysis of gene expression and microRNA expression by real-time RT-PCR, flow cytometric analysis, soft agar assay. See Supplementary Methods for details.

\section{Statistical analysis}

Results were expressed as mean value $\pm \mathrm{SD}$ and interpreted by one-way ANOVA. Differences were considered statistically significant when $P<0.05$.

\section{ACKNOWLEDGMENTS}

We thank professor Hong-Liang Li (Cardiovascular Research Institute of Wuhan University) for B6.FVB(Cg)Mmp $9^{t m I T v u / J}$ mice; Zhi-Hui Liang, Hui-Fen Zhu, ShengHong Liu for technical assistance; professor Guan-Xin Shen, Xin-Xing Wu, Zhan-Qiu Yang for comments on the manuscript.

\section{CONFLICTS OF INTEREST}

The authors declare that there are no conflicts of interest to disclose.

\section{GRANT SUPPORT}

This work was supported by National Natural Science Foundation of China (No. 81472704, 81272314,
30830095), and National Development Program (973) For

Key Basic Research of China (No. 2009CB521806).

\section{REFERENCES}

1. Goss PE, Chambers AF. Does tumour dormancy offer a therapeutic target? Nat Rev Cancer. 2010; 10:871-877.

2. Páez D, Labonte MJ, Bohanes P, Zhang W, Benhanim L, Ning Y, Wakatsuki T, Loupakis F, Lenz HJ. Cancer dormancy: a model of early dissemination and late cancer recurrence. Clin Cancer Res. 2012; 18:645-653.

3. Zappalà G, McDonald PG, Cole SW. Tumor dormancy and the neuroendocrine system: an undisclosed connection? Cancer Metastasis Rev. 2013; 32:189-200.

4. Alizadeh AM, Shiri S, Farsinejad S. Metastasis review: from bench to bedside. Tumour Biol. 2014; 35:8483-8523.

5. Aguirre-Ghiso JA. Models, mechanisms and clinical evidence for cancer dormancy. Nat Rev Cancer. 2007; $7: 834-846$

6. Jablonska J, Leschner S, Westphal K, Lienenklaus S, Weiss S. Neutrophils responsive to endogenous IFN-beta regulate tumor angiogenesis and growth in a mouse tumor model. J Clin Invest. 2010; 120:1151-1164.

7. Tazzyman S, Lewis CE, Murdoch C. Neutrophils: key mediators of tumour angiogenesis. Int J Exp Pathol. 2009; 90:222-231.

8. Nozawa H, Chiu C, Hanahan D. Infiltrating neutrophils mediate the initial angiogenic switch in a mouse model of multistage carcinogenesis. Proc Natl Acad Sci U S A. 2006; 103:12493-12498.

9. Wu CF, Andzinski L, Kasnitz N, Kröger A, Klawonn F, Lienenklaus S, Weiss S, Jablonska J. The lack of type I interferon induces neutrophil-mediated pre-metastatic niche formation in the mouse lung. Int J Cancer. 2015;137:837-847.

10. Wculek SK, Malanchi I. Neutrophils support lung colonization of metastasis-initiating breast cancer cells. Nature. 2015; 528:413-417.

11. Lavazza C, Carlo-Stella C, Giacomini A, Cleris L, Righi M, Sia D, Di Nicola M, Magni M, Longoni P, Milanesi M, Francolini M, Gloghini A, Carbone A, et al. Human CD34 ${ }^{+}$ cells engineered to express membrane-bound tumor necrosis factor-related apoptosis-inducing ligand target both tumor cells and tumor vasculature. Blood. 2010; 115:2231-2240.

12. Yan B, Wei JJ, Yuan Y, Sun R, Li D, Luo J, Liao SJ, Zhou YH, Shu Y, Wang Q, Zhang GM, Feng ZH. IL-6 cooperates with G-CSF to induce protumor function of neutrophils in bone marrow by enhancing STAT3 activation. J Immunol. 2013; 190:5882-5893.

13. Mishalian I, Bayuh R, Levy L, Zolotarov L, Michaeli J, Fridlender ZG. Tumor-associated neutrophils (TAN) develop pro-tumorigenic properties during tumor progression. Cancer Immunol Immunother. 2013; 62:1745-1756. 
14. Panigrahy D, Edin ML, Lee CR, Huang S, Bielenberg DR, Butterfield CE, Barnés CM, Mammoto A, Mammoto T, Luria A, Benny O, Chaponis DM, Dudley AC, et al. Epoxyeicosanoids stimulate multiorgan metastasis and tumor dormancy escape in mice. J Clin Invest. 2012; 122:178-191.

15. Wang D, Dubois RN. Epoxyeicosatrienoic acids: a doubleedged sword in cardiovascular diseases and cancer. J Clin Invest. 2012; 122:19-22.

16. Jiang JG, Ning YG, Chen C, Ma D, Liu ZJ, Yang S, Zhou J, Xiao X, Zhang XA, Edin ML, Card JW, Wang J, Zeldin DC, et al. Cytochrome p450 epoxygenase promotes human cancer metastasis. Cancer Res. 2007; 67:6665-6674.

17. Cheranov SY, Karpurapu M, Wang D, Zhang B, Venema RC, Rao GN. An essential role for SRC-activated STAT-3 in 14,15-EET-induced VEGF expression and angiogenesis. Blood. 2008; 111:5581-5591.

18. Zhang B, Cao H, Rao GN. Fibroblast growth factor-2 is a downstream mediator of phosphatidylinositol 3-kinaseAkt signaling in 14,15-epoxyeicosatrienoic acid-induced angiogenesis. J Biol Chem. 2006; 281:905-914.

19. Feng XX, Liu M, Yan W, Zhou ZZ, Xia YJ, Tu W, Li PY, Tian DA. $\beta 3$ integrin promotes TGF- $\beta 1 / \mathrm{H}_{2} \mathrm{O}_{2} /$ HOCl-mediated induction of metastatic phenotype of hepatocellular carcinoma cells by enhancing TGF- $\beta 1$ signaling. PLoS One. 2013; 8:e79857.

20. Zhou YH, Liao SJ, Li D, Luo J, Wei JJ, Yan B, Sun R, Shu Y, Wang Q, Zhang GM, Feng ZH. TLR4 ligand/ $\mathrm{H}_{2} \mathrm{O}_{2}$ enhances TGF- $\beta 1$ signaling to induce metastatic potential of non-invasive breast cancer cells by activating non-Smad pathways. PLoS One. 2013; 8:e65906.

21. Kolaczkowska E, Kubes P. Neutrophil recruitment and function in health and inflammation. Nat Rev Immunol. 2013; 13:159-175.

22. Kobayashi Y. The role of chemokines in neutrophil biology. Front Biosci. 2008; 13:2400-2407.

23. Fridlender ZG, Sun J, Kim S, Kapoor V, Cheng G, Ling L, Worthen GS, Albelda SM. Polarization of tumor-associated neutrophil phenotype by TGF-beta: „N1“ versus „N2“ TAN. Cancer Cell. 2009; 16:183-194.

24. Node K, Huo Y, Ruan X, Yang B, Spiecker M, Ley K, Zeldin DC, Liao JK. Anti-inflammatory properties of cytochrome P450 epoxygenase-derived eicosanoids. Science. 1999; 285:1276-1279.

25. Saatian B, Zhao Y, He D, Georas SN, Watkins T, Spannhake EW, Natarajan V. Transcriptional regulation of lysophosphatidic acid-induced interleukin-8 expression and secretion by p38 MAPK and JNK in human bronchial epithelial cells. Biochem J. 2006; 393:657-668.

26. Kim YM, Reed W, Wu W, Bromberg PA, Graves LM, Samet JM. $\mathrm{Zn}^{2+}$-induced IL-8 expression involves AP-1, JNK, and ERK activities in human airway epithelial cells. Am J Physiol Lung Cell Mol Physiol. 2006; 290: L1028-1035.
27. Yeh M, Gharavi NM, Choi J, Hsieh X, Reed E, Mouillesseaux KP, et a. Oxidized phospholipids increase interleukin 8 (IL-8) synthesis by activation of the c-src/ signal transducers and activators of transcription (STAT)3 pathway. J Biol Chem. 2004; 279:30175-30181.

28. Wu T, Wang Z, Liu Y, Mei Z, Wang G, Liang Z, Cui A, Hu X, Cui L, Yang Y, Liu CY. Interleukin 22 protects colorectal cancer cells from chemotherapy by activating the STAT3 pathway and inducing autocrine expression of interleukin 8. Clin Immunol. 2014; 154:116-126.

29. Chuang TD, Luo X, Panda H, Chegini N. miR-93/106b and their host gene, MCM7, are differentially expressed in leiomyomas and functionally target F3 and IL-8. Mol Endocrinol. 2012; 26:1028-1042.

30. Wei $\mathrm{T}, \mathrm{Xu} \mathrm{N}$, Meisgen F, Ståhle M, Sonkoly E, Pivarcsi A. Interleukin- 8 is regulated by miR-203 at the posttranscriptional level in primary human keratinocytes. Eur J Dermatol. 2013. [Epub ahead of print].

31. Goretti E, Rolland-Turner M, Léonard F, Zhang L, Wagner DR, Devaux Y. MicroRNA-16 affects key functions of human endothelial progenitor cells. J Leukoc Biol. 2013; 93:645-655.

32. Xu N, Meisgen F, Butler LM, Han G, Wang XJ, SöderbergNauclér C, Ståhle M, Pivarcsi A, Sonkoly E. MicroRNA-31 is overexpressed in psoriasis and modulates inflammatory cytokine and chemokine production in keratinocytes via targeting serine/threonine kinase 40. J Immunol. 2013; 190:678-688.

33. Kuo PL, Liao SH, Hung JY, Huang MS, Hsu YL. MicroRNA-33a functions as a bone metastasis suppressor in lung cancer by targeting parathyroid hormone related protein. Biochim Biophys Acta. 2013; 1830:3756-3766.

34. Perng DW, Yang DM, Hsiao YH, Lo T, Lee OK, Wu MT, $\mathrm{Wu}$ YC, Lee YC. miRNA-146a expression positively regulates tumor necrosis factor- $\alpha$-induced interleukin- 8 production in mesenchymal stem cells and differentiated lung epithelial-like cells. Tissue Eng Part A. 2012; 18:2259-2267.

35. Kuo YC, Li YS, Zhou J, Shih YR, Miller M, Broide D, Lee OK, Chien S. Human mesenchymal stem cells suppress the stretch-induced inflammatory miR-155 and cytokines in bronchial epithelial cells. PLoS One. 2013; 8:e71342.

36. Huang L, Liu Y, Wang L, Chen R, Ge W, Lin Z, Zhang Y, Liu S, Shan Y, Lin Q, Jiang M. Down-regulation of miR-301a suppresses pro-inflammatory cytokines in Toll-like receptortriggered macrophages. Immunology. 2013;140:314-322.

37. Marsolier J, Pineau S, Medjkane S, Perichon M, Yin Q, Flemington E, Weitzman MD, Weitzman JB. OncomiR addiction is generated by a miR-155 feedback loop in Theileria-transformed leukocytes. PLoS Pathog. 2013; 9:e1003222.

38. Schmeck B, Moog K, Zahlten J, van Laak V, N`Guessan PD, Opitz B, Rosseau S, Suttorp N, Hippenstiel S. Streptococcus pneumoniae induced c-Jun-N-terminal kinase- and AP-1- 
dependent IL-8 release by lung epithelial BEAS-2B cells. Respir Res. 2006; 7:98.

39. Yin Q, Wang X, McBride J, Fewell C, Flemington E. $\mathrm{B}$-cell receptor activation induces $\mathrm{BIC} / \mathrm{miR}-155$ expression through a conserved AP-1 element. J Biol Chem. 2008; 283:2654-2662.

40. Granot Z, Henke E, Comen EA, King TA, Norton L, Benezra R. Tumor entrained neutrophils inhibit seeding in the premetastatic lung. Cancer Cell. 2011; 20:300-314.

41. Cetean S, Căinap C, Constantin AM, Căinap S, Gherman A, Oprean L, Hangan A, Oprean R. The importance of the granulocyte-colony stimulating factor in oncology. Clujul Med. 2015; 88:468-472.

42. Ataie-Kachoie P, Pourgholami MH, Richardson DR, Morris DL. Gene of the month: Interleukin 6 (IL-6). J Clin Pathol. 2014; 67:932-937.

43. Pozzi A, Macias-Perez I, Abair T, Wei S, Su Y, Zent R, Falck JR, Capdevila JH. Characterization of 5,6- and 8,9-epoxyeicosatrienoic acids (5,6- and 8,9-EET) as potent in vivo angiogenic lipids. J Biol Chem. 2005; 280:2713827146

44. Ardi VC, Kupriyanova TA, Deryugina EI, Quigley JP. Human neutrophils uniquely release TIMP-free MMP-9 to provide a potent catalytic stimulator of angiogenesis. Proc Natl Acad Sci U S A. 2007; 104:20262-20267.
45. Zeldin DC. Epoxygenase pathways of arachidonic acid metabolism. J Biol Chem. 2001; 276:36059-36062

46. Shin JS, Hong Y, Lee HH, Ryu B, Cho YW, Kim NJ, Jang DS, Lee KT. Fulgidic Acid Isolated from the Rhizomes of Cyperus rotundus Suppresses LPS-Induced iNOS, COX-2, TNF- $\alpha$, and IL- 6 Expression by AP-1 Inactivation in RAW264.7 Macrophages. Biol Pharm Bull. 2015; 38:1081-1086.

47. Wang D, Wang H, Brown J, Daikoku T, Ning W, Shi Q, Richmond A, Strieter R, Dey SK, DuBois RN. CXCL1 induced by prostaglandin E2 promotes angiogenesis in colorectal cancer. J Exp Med. 2006; 203:941-951.

48. Daley JM, Thomay AA, Connolly MD, Reichner JS, Albina JE. Use of Ly6G-specific monoclonal antibody to deplete neutrophils in mice. J Leukoc Biol. 2008; 83:64-70.

49. Liu Y, Huang B, Yuan Y, Gong W, Xiao H, Li D, Yu ZR, Wu FH, Zhang GM, Feng ZH. Inhibition of hepatocarcinoma and tumor metastasis to liver by gene therapy with recombinant CBD-HepII polypeptide of fibronectin. Int J Cancer. 2007; 121:184-192.

50. Gong W, Zhang GM, Liu Y, Lei Z, Li D, Yuan Y, Huang B, Feng ZH. IFN-gamma withdrawal after immunotherapy potentiates B16 melanoma invasion and metastasis by intensifying tumor integrin alphavbeta3 signaling. Int $\mathrm{J}$ Cancer. 2008; 123:702-708. 